\title{
Assessment of the Effectiveness and Safety of Endotracheal Intubation for Inhalational Anesthesia without the Use of Muscle Relaxants or Analgesics in Adults
}

\author{
Berrocal AR ${ }^{1 *}$, Perez $\mathrm{LR}^{2}$ and Fernandez $\mathrm{JG}^{3}$ \\ ${ }^{1}$ Consultant, Anaesthesiology and Resuscitation Service, University Hospital Puerta de \\ Hierro Majadahonda, Spain
}

${ }^{2}$ Consultant, Neuro surgery Service, University Hospital Puerta de Hierro Majadahonda, Spain

${ }^{3}$ Head of the Anesthesiology and resuscitation service at the Puerta de Hierro-Majadahonda hospital, Spain

*Corresponding author: Antonio Romero Berrocal, M.D, PhD. Consultant. Anaesthesiology and Resuscitation Service. University Hospital Puerta de Hierro Majadahonda, C/Manzano no19, Rivasvaciamadrid, 28522, Madrid, Spain, Tel: +34687403747; Email: antonromero@hotmail.com

\section{Abstract}

Background: Tracheal intubation is achieved with drugs given for the induction of general anesthesia. This process is facilitated by administering a muscle relaxant. However, administration of muscle relaxants may be associated with side effects. The necessity to avoid the use of muscle relaxants becomes imperative in patients who are allergic to neuromuscular relaxants, or with difficult airway. The rationale for tracheal intubation without muscle relaxants may not be obvious to many health care providers in adults. Avoiding muscle relaxants can prevent the potential complications of their use and a faster return of spontaneous ventilation may be evidenced. Anesthesia inducction with gas may be an alternative to a safe and efficient orotracheal intubation without muscle relaxants and analgesic drugs. The aim of this study was to identify complications and evaluate the efficacy of pure inhalational anesthesia induction to achieve endotracheal intubation without the use of muscle relaxant and analgesic drugs.

Methods: Prospective and randomiced study in 91 patients. Anesthesia induction was performed, without analgesics and muscle relaxants. The optimal intubation conditions were considered when the laryngoscopy showed the glottis completely open.

Results: Orotracheal intubation was performed in 100\%. The mean time lapsed until optimal conditions for intubation were achieved was $6 \pm 0.88$ minutes. The mean sevoflurane end-tidal volume at which intubation could be performed was $5.5 \% \pm 0.26 \%$.Transitory apnea in 57.1\%, and apnea in $1.1 \%$. Nausea and vomiting was $6.6 \%$. 


\section{Anaesthesia \& Critical Care Medicine Journal}

Conclusions: the use of pure inhalational induction is an effective and safe technique to perform endotracheal intubation.

Keywords: Adults Anesthesia Inhalation Relaxants

Abbreviations: LVEF: Left ventricle ejection fraction; FGF: Fresh gas flow; ASA: American Society of Anesthesiologists; OTT: Orotracheal tube; MAP: Mean arterial pressure; PONV: Postoperative nausea and vomiting; SD: Standard deviation; OTI: Orotracheal intubation; LMA: Laryngeal mask.

\section{Plain Languaje Summary}

During the induction of hypnosis during general anesthesia in adults, muscle relaxants and analgesics can be used. This study shows the possibility of performing this induction effectively and safely using only inhalation gas. It is an alternative for patients allergic to these drugs or when we want to avoid apnea during induction.

\section{Introduction}

Tracheal intubation is achieved with drugs given for the induction of general anesthesia such as propofol and fentanyl, and in addition, this process is often facilitated by administering a muscle relaxant drug. However, administration of depolarizing muscle relaxants such as succinylcholine may be associated with side effects, ranging from postoperative myalgias to a more severe side effects such as malignant hyperthermia or increased intracranial pressure $[1,2]$. The necessity to avoid the use of muscle relaxants during anesthesia becomes imperative in patients who are allergic to neuromuscular relaxants, or in case of difficult airway to avoid the patient apnea. The rationale for tracheal intubation without muscle relaxants (referred as pure inhalational induction in this manuscript) may not be obvious to many health care providers. Nevertheless, avoiding muscle relaxants can prevent the potential complications of their use. Additionally, a faster return of spontaneous ventilation may be evidenced when fentanyl and propofol are used for induction [3]. Given the hypnotic, analgesic and muscle relaxant effect of sevoflurane, induction of anesthesia with this gas may be an alternative to a safe and efficient orotracheal intubation without the use of muscle relaxants and/or analgesic drugs [4]. The aim of this study was to identify complications and evaluate the efficacy of pure inhalational anesthesia induction to achieve endotracheal intubation without the use of muscle relaxant and analgesic drugs.

\section{Methods}

This study was registered at ClinicalTrials.gov on April 13, 2017 with the Identifier: NCT03112564. Name of the main author: Antonio Romero Berrocal This is a prospective study conducted between March 2013 and November 2014 at Hospital Universitario Puerta de Hierro-Majadahonda, in Madrid, Spain, with the previous approval by the institutional clinical research ethics committee, and written informed consent was obtained from all subjects. A total of 91 subjects who underwent general, gastrointestinal, orthopedic, urology and neurological surgery were randomly selected and provided written informed consent before any studyrelated procedures were performed. Subjects were randomly selected and Study inclusion criteria consisted in women and men of legal age (18 years) ranging from 23 to 87 years. ASA I, II and III. Subjects with left ventricle ejection fraction (LVEF) of $<35 \%$, history of ischemic heart disease in the last year, history of malignant hyperthermia, presence of thoracic drains, increased intracranial pressure or brain tumors undergoing neurophysiological monitoring, and subjects with hemodynamic instability or likely to become unstable during induction of anesthesia were excluded from the study. A Dräger Primus ${ }^{\circledR}$ brand anesthesia workstation was used. Anesthetic circuit with sevoflurane $8 \%$ with fresh gas flow (FGF) of $10 \mathrm{~L} / \mathrm{min}$, and cycling for 20 seconds was previously primed. Anesthesia induction was performed at tidal volume, avoiding the use of analgesics and/or muscle relaxants with FGF of $6 \mathrm{~L} / \mathrm{min}$. Induction time was shortened as the FGF was increased. Once 5\% sevoflurane end-tidal volume was reached, ventilation with facial mask was maintained for three more minutes. The following parameters were recorded in each participant: age, sex, American Society of Anesthesiologists (ASA) level, home medications, time lapsed until the loss of blink reflex was achieved and orotracheal tube (OTT) was placed, difficulties in OTT placement, sevoflurane end-tidal volume after OTT placement, complications related to OTT insertion (movement, coughing, rigidity, apnea), mean arterial 


\section{Anaesthesia \& Critical Care Medicine Journal}

pressure (MAP) variations, subject's satisfaction following surgery ( a questionnaire was made to each patient in which they were asked if they were satisfied with the anesthetic procedure and they had to choose between Nothing satisfied, Not satisfied, Satisfied), and postoperative nausea and vomiting (PONV) during the first 12 hours after surgery. The optimal intubation conditions were considered when the laryngoscopy showed the glottis completely open. During the induction, we performed laryngoscopy every 30 seconds after minute [4]. Once the subject was successfully intubated, analgesia was provided with fentanyl $2 \mathrm{mcg} / \mathrm{kg}$. No muscle relaxants were given during surgery. Maintenance of anesthesia was achieved with sevoflurane, until reaching a $\mathrm{MAC}$ of 1.3 , and fentanyl $4-6 \mathrm{mg} / \mathrm{kg}$ was given as needed for the duration of the surgical procedure. PONV prophylaxis was performed with $8 \mathrm{mg}$ of intravenous (IV) dexamethasone at the beginning of surgery, and ondansetron $4 \mathrm{mg}$ IV at the end of surgery, independently of the preoperative Apfel score for PONV risk stratification.

\section{Statistical Analysis}

Quantitative variables were expressed as mean \pm standard deviation (SD), or as median (interquartile range) in the absence of Gaussian distribution, and these were compared using the T-student test, or the nonparametric test of Mann-Whitney respectively. Qualitative variables were expressed as percentages, and were compared using the Chi-square $(\chi 2)$ test. For ordinal variables, the hypothesis of linear tendency of proportions was contrasted. $\mathrm{P}$ values of $<0.05$ were considered statistically significant. For the statistical analysis, statistical pack SPSS v14.0 was used.

\section{Results}

Orotracheal intubation (OTI) was performed in 100\% of the subjects without the use of muscle relaxants. A Cook® Medical Frova tracheal tube introducer was needed in 5 subjects to achieve OTI. The average time until blink reflex disappeared was $40.9 \pm 5.57$ seconds, and the mean time lapsed until optimal conditions for intubation were achieved was $6 \pm 0.88$ minutes. The mean sevoflurane end-tidal volume at which intubation could be performed was $5.5 \% \pm 0.26 \%$. Difficulty in intubation was experienced in 2 subjects, in whom a higher hypnotic depth was required to achieve laryngeal opening. The mean drop in MAP during intubation was $16.1 \% \pm 8.85 \%$ (CI 95\%), $p<0.001$. Cough and rigidity (clinical approximation) was evidenced in $16.5 \%$ and $6.6 \%$ of subjects respectively. Transitory apnea ( $<20$ seconds) was reported in $57.1 \%$ of subjects, and apnea for $>20$ seconds in $1.1 \%$ of subjects in whom ventilator assistance was required. The incidence of nausea and vomiting during the first three postoperative hours was $6.6 \%$ of subjects. Laryngospasm was not reported in any case, and movement during induction was evidenced in $15 \%$ of the subjects. Subject satisfaction was $100 \%$. All of the above mentioned complications were not related to sex or ASA level, except rigidity that was associated in subjects with ASA III (Table 1).

\begin{tabular}{|c|c|}
\hline $\begin{array}{c}\text { Average time until blink reflex } \\
\text { disappeared }\end{array}$ & $\begin{array}{c}40.9 \pm 5.57 \\
\text { seconds }\end{array}$ \\
\hline $\begin{array}{c}\text { Mean time lapsed until optimal } \\
\text { conditions for intubation }\end{array}$ & $6 \pm 0.88$ minutes \\
\hline $\begin{array}{c}\text { Sevoflurane end-tidal at which } \\
\text { intubation could be performed }\end{array}$ & $5.5 \% \pm 0.26$ \\
\hline Transitory apnea (<20 seconds) & $57.10 \%$ \\
\hline $\begin{array}{c}\text { Nausea and vomiting during the first } \\
\text { 3 postoperative hours }\end{array}$ & $6.60 \%$ \\
\hline Cough & $16.50 \%$ \\
\hline Rigidity(clinical appreciation) & $6.60 \%$ \\
\hline
\end{tabular}

Table 1: Results

\section{Discussion}

The results of the present study suggest that anesthesia induction with sevoflurane alone provides good or excellent conditions for tracheal intubation in most patients. This may be possible due to the hypnotic, analgesic and muscle relaxant effects of this gas [4]. The mean time from onset of apnea at the induction of anesthesia until conditions to achieve tracheal intubation were met was less than 7 minutes in all subjects.

The major deficiency in the study design was the failure to include a control group (induction with standard anesthesia regimen that includes administration of muscle relaxants and propofol). Evading the side effects of anesthesia that may delay patient discharge or their return to daily activities such as postoperative myalgia, nausea and vomiting, is an important goal to achieve in all surgical procedures. This can be achieved avoiding the administration of muscle relaxants when they are not required for the planned procedure3. For example, health care providers might avoid the use of succinylcholine, and thus sidestep its side effects including the risk of 


\section{Anaesthesia \& Critical Care Medicine Journal}

malignant hyperthermia and arrhythmias [5]. Moreover, when non-depolarizing muscle relaxants are not administered, the complications associated with their antagonism (increased risk of PONV) are also avoided. Nevertheless, tracheal intubation without the use of neuromuscular blockade agents may cause harm if attempted under inadequate conditions and cause trauma to the airway or ventilation can be result inadequate [3]. In the present study, OTI was achieved without the use of muscle relaxants and analgesics, representing an important advantage in anesthesia induction in patients with probable or known difficult airway intubation. The intention of this study was to maintain spontaneous ventilation, an adequate level of unconsciousness, and achieve optimal conditions for intubation using inhalational gas as the only therapy.

The use of anesthesia induction with inhalational agents in adults has been described in the habitual anesthesia practice, and has also been accepted as an efficacious and safe technique for the insertion of laryngeal mask [6-8]. The rapidness by which subjects in the present study were unconscious during pure inhalational induction is similar to other studies that utilized the same FGF of $6 \mathrm{~L} / \mathrm{min} 8$. Till the date, a variety of studies comparing the conditions for the insertion of laryngeal mask (LMA) through inhalational induction with sevoflurane or with IV propofol had been published $[4,9]$; however, there is a lack of studies describing the same technique for the insertion of endotracheal tube in adults.

In general, studies including adult and elderly subjects have been able to show significant hemodynamic changes during induction with sevoflurane $5 \%$ and using the endtidal volume induction method for the placement of LMA, and that it can be an alternative to induction with propofol. In a randomized, double-blinded study conducted by Walpole et al, the authors concluded that sevoflurane decreases blood pressure in less extent than propofol during pure inhalational induction [10]. In another randomized controlled trial conducted by Vidal, et al. [11]. The authors demonstrated the efficacy, side effects, and hemodynamic alterations during the anesthetic induction with sevoflurane in 30 subjects scheduled to undergo coronary revascularization surgery, comparing techniques of tidal volume and vital capacity. The authors concluded that hemodynamic stability seems to be similar when providing anesthetic induction with inhaled sevoflurane with both techniques, and that MAP decreased in both groups without significant differences. The results of our study agree with these publications, and it proves that inhalational induction technique is possible and safe for adult subjects. In our study, the drop in MAP during induction was inferior to that described by Kirkbride, et al. [6], in which MAP dropped $22 \pm 13 \%$ after induction with sevoflurane $8 \%$. Thwaites et al. 7 observed MAP decreases of $10 \mathrm{mmHg}$ during inhalational induction with sevoflurane, and also reported the occurrence of cough in $8 \%$ of subjects undergoing inhalational induction. This lower incidence of cough in this study could be justified by the administration of intravenous lidocaine previous induction to ameliorate the pain caused by propofol during induction. Sevoflurane 8\% induces a more frequent and longer apnea when compared to lower concentrations6. Siddik, et al. [12]. In a series of 26 subjects who underwent inhalational induction with $8 \%$ sevoflurane, found a $7 \%$ incidence of apnea with a mean duration of 27 seconds; this result could be justified by the higher gas concentration used. In a meta-analysis by Joo, et al. [4]. Comparing the use of propofol vs sevoflurane for the induction of anesthesia, a lower incidence of apnea was observed. This finding is higher than the one observed in our study. The incidence of postoperative nausea and vomiting is about $20-30 \%$ in general population, and up to $80 \%$ in high risk subjects. The risk factors depend on the anesthetic technique, type of surgery and factors that depend on patient characteristics. The risk is stratified considering the following factors: gender, smoking status, history of PONV, and use of opioids for postoperative analgesia [13]. In our study, the incidence of PONV was approximately of $6 \%$ after double antiemetic prophylaxis was administered in all subjects. This is within the expected incidence for PONV in patients who received dual prophylaxis described in the literature [13]. Satisfaction with the technique used in our study was high $(100 \%)$, consistent with previous research that report satisfaction rates of $>$ $90 \%[6,14]$.

\section{Conclusion}

Based on the results of our study and previous literature, the authors conclude that the use of pure inhalational induction is an effective and safe technique to perform endotracheal intubation, and it may be used as an alternative to the administration of muscle relaxants and analgesics especially in patients who this type of drugs are contraindicated, or to assess the airway in a possible difficult airway. The authors recommend stratification of the patients for the use of this technique in order to stablish the adequate scenario to perform it in a safely and effective manner and with the minimal incidence of complications. 


\section{Anaesthesia \& Critical Care Medicine Journal}

\section{References}

1. Scheller MS, Zornow MH, Saidman LJ (1992) Tracheal Intubation Without the Use of Muscle Relaxants: A Technique Using Propofol and Varying Doses of Alfentanil. Anesthesia \& Analgesia 75(5): 788-793.

2. Savarese JJ, Caldwell JE, Lien CA, Miller RD (2000) Pharmacology of muscle relaxants and their antagonists. Anesthesia 5: 412-490.

3. Stevens JB, Wheatley L (1998) Tracheal intubation in ambulatory surgery patients: using remifentanil and propofol without muscle relaxants. Anesthesia \& Analgesia 86(1): 45-49.

4. Joo HS, Perks WJ (2000) Sevoflurane versus propofol for anesthetic induction: a meta-analysis. Anesth Analg 91(1): 213-219.

5. Trépanier CA, Brousseau C, Laccrte L (1988) Myalgia in outpatient surgery: comparison of atracurium and succinylcholine. Canadian journal of anaesthesia 35(3): 255-258.

6. Kirkbride DA, Parker JL, Williams GD, Buggy DJ (2001) Induction of anesthesia in the elderly ambulatory patient: a double-blinded comparison of propofol and sevoflurane. Anesth Analg 93(5): 11851187.

7. Thwaites A, Edmends S, Smith I (1997) Inhalation induction with sevoflurane: a double-blind comparison with propofol. British Journal of anaesthesia 78(4): 356-361.

8. Knaggs C, Drummond G (2005) Randomized comparison of three methods of induction of anaesthesia with sevoflurane. British journal of anaesthesia 95(2): 178-182.

9. Topuz D, Postaci A, Sacan O, Yildiz N, Dikmen B (2010) A comparison of sevoflurane induction versus propofol induction for laryngeal mask airway insertion in elderly patients. Saudi medical journal 31(10): 1124-1129.

10. Walpole R, Logan M (1999) Effect of sevoflurane concentration on inhalation induction of anaesthesia in the elderly. British journal of anaesthesia 82(1): 20-24.

11. Vidal MA, Calderon E, Martinez E, Pernia A, Torres LM (2006) Comparison of 2 techniques for inhaled anesthetic induction with sevoflurane in coronary artery revascularization. Rev Esp Anestesiol Reanim 53(10): 639-642.

12. Siddik-Sayyid SM, Aouad MT, Taha SK, Daaboul DG, Deeb PG, et al. (2005) A comparison of sevofluranepropofol versus sevoflurane or propofol for laryngeal mask airway insertion in adults. Anesth Analg 100(4): 1204-1209.

13. Marcoval IB, Cerrillo PG (2006) Estratificación del riesgo, profilaxis y tratamiento de las náuseas y vómitos postoperatorios. Rev Esp Anestesiol Reanim 53(5): 301-311.

14. Philip BK, Lombard LL, Roaf ER, Drager LR, Calalang I, et al. (1999) Comparison of vital capacity induction with sevoflurane to intravenous induction with propofol for adult ambulatory anesthesia. Anesth Analg 89(3): 623-627. 